\title{
Chlamydial infection of the urethra in men
}

\author{
H. M. PERROUD AND K. MIEDZYBRODZKA \\ From the Department of Dermato-Venereology, University of Lausanne, Switzerland
}

SUMMARY Chlamydia trachomatis was isolated from the urethra of $124(52 \%)$ of 238 men with non-gonococcal urethritis (NGU). Repeat isolation attempts in 155 of these patients were successful in eight men in whom results had been negative on the initial visit, but they were unsuccessful in eight men who initially had had positive cultures. We must assume that with our present isolation techniques we are missing, at any single visit, at least $9 \%$ of chlamydial infections. $C$. trachomatis was also found in $32(23 \%)$ of 139 men with gonorrhoea. Positive cultures were obtained from 15 $(79 \%)$ of 19 men, who later developed post-gonococcal urethritis (PGU). Thiamphenicol, used for the treatment of gonorrhoea, was shown to have very little effect on $C$. trachomatis, which could still be recovered after treatment in $76 \%$ of the patients who initially had had a combined infection. The typing of 35 genital isolates by micro-immunofluorescence confirms the previously reported distribution of chlamydial serotypes. In this study a social profile is given of our patients with urethritis and a comparison is made of the duration of symptoms and the nature of discharge in men with gonococcal, chlamydial, and non-specific urethritis. We were able to show a clear difference in clinical symptoms in men with gonorrhoea and NGU, taken as a whole, but found only a slight difference between men with chlamydial and non-specific urethritis.

\section{Introduction}

The incidence of non-gonococcal urethritis (NGU) has risen more rapidly than that of gonorrhoea in England (Department of Health and Social Security, 1976), Sweden (Juhlin, 1975), and the USA (McChesney et al., 1973) and it is now recognised as the most common sexually transmitted disease. Although no statistics are available as NGU is not reported, data from the Department of DermatoVenereology in Lausanne suggest that this trend also applies to Switzerland (Perroud, 1977). NGU accounted for $55 \%$ of all our cases of urethritis in 1976. Chlamydia trachomatis has been incriminated as one of the most common sexually transmitted genital pathogens, and is reported to be associated with approximately half of all cases of NGU. Simplified cell culture techniques can now isolate C. trachomatis and make it possible to carry out larger epidemiological studies. We hoped to determine the incidence of chlamydial infections in
Address for reprints: H. M. Perroud, Department of DermatoVenereology, CHUV, 1010 Lausanne, Switzerland

Received for publication 8 July 1977 patients presenting with urethritis and to define more clearly their clinical characteristics in comparison with gonococcal and non-specific urethritis (NSU).

\section{Materials and methods}

SELECTION OF CASES AND DIAGNOSTIC CRITER I A

The patients, all men, were examined between March 1975 and April 1977 in the Department of Dermato-Venereology of the University Hospital in Lausanne. All had presented with the complaint of urethral discharge with or without dysuria. A standard medical history was taken for each patient concerning symptoms and findings, previous genital infections, and sexual contacts. The clinical assessment included collecting secretions for bacteriological examination after gently stripping the anterior urethra. When no exudate could be obtained by this method, prostatic massage was performed.

\section{GONORRHOEA}

Urethral smears showing Gram-negative intracellular diplococci were considered as presumptive 
evidence and confirmed in all cases by a positive culture for Neisseria gonorrhoeae. Cultures were made on Thayer Martin selective culture medium (Baltimore Biological Laboratories).

\section{NON-GONOCOCCAL URETHRITIS}

Only cases with visible discharge, either spontaneously or after gentle urethral massage, and more than 20 leucocytes on at least three fields examined at $\times 400$ magnification were included. Gonorrhoea was excluded by a negative smear result and by at least one negative culture; two culture attempts were performed in most cases. Trichomonas vaginalis infections were excluded by wet films.

\section{POST-GONOCOCCAL URETHRITIS}

Post-gonococcal urethritis (PGU) was diagnosed if the patient still had a discharge seven or more days after treatment for gonorrhoea, or if a discharge appeared on gentle urethral massage, smears and cultures were negative for gonococci and trichomonads, but the microscopical criteria for NGU were fulfilled.

\section{CONTROL GROUP}

Men included in this group had neither a history nor symptoms of urethritis and matched the groups with gonococcal and non-gonococcal infections in regard to age, marital state, percentage of homosexuals, and number of partners. Twelve of the controls were non-infected contacts of women with gonorrhoea, trichomoniasis, or candidosis; 14 had genital conditions other than urethritis, 10 were students or staff, and four men requested examination but nothing was found.

\section{CRITERIA FOR EXCLUSION}

Patients who had received antibiotics during the two weeks before the initial visit were not included in the study. A few patients with gonorrhoea and NGU had to be excluded because inoculation of the cultures was delayed for more than 24 hours, or because contamination of the cell cultures rendered the isolation of $C$. trachomatis impossible. No other criteria were used to divide the patients into groups.

\section{ISOLATION OF C. TRACHOMATIS \\ Collection of specimens}

The method of Reeve et al. (1975) was used to collect specimens and isolate $C$. trachomatis. Urethral specimens were collected by inserting a calcium-alginate-tipped wire swab $3-4 \mathrm{~cm}$ into the urethra. The tip was then cut into a vial containing $2 \mathrm{ml}$ of transport medium. This transport medium consisted of growth medium (see below) supplemented with glucose $1 \%$ by vol. of stock $(3 \mathrm{~mol} / \mathrm{l})$ and amphotericin B $2.5 \mu \mathrm{g} / \mathrm{ml}$. The collected specimens were kept at a temperature of at least $4^{\circ} \mathrm{C}$ and isolation was attempted within 24 hours.

\section{Cell cultures}

McCoy cells were grown in Eagle's minimal essential medium supplemented with heat inactivated fetal calf serum $10 \%$ by vol., glutamine $1 \%$ by vol. of stock $(30 \mathrm{mg} / \mathrm{ml}$ ), and vitamins (Flow lab. $100 \times$ concentrate) to which was added gentamicin $0 \cdot 1$ $\mathrm{mg} / \mathrm{ml}$. When confluent, the cells were trypsinised and suspended at $1 \times 10^{5}$ cells $/ \mathrm{ml}$ of growth medium to which was added 5-iodo-2'deoxyuridine (IUDR) $25 \mu \mathrm{g} / \mathrm{ml}$. One millilitre of this suspension was placed in each $10 \mathrm{ml}$ flat-bottomed vial containing a $12 \mathrm{~mm}$ diameter coverslip. After incubation at $35^{\circ} \mathrm{C}$ for three days the monolayers were ready for chlamydial isolation and were used for up to seven days after seeding.

\section{ISOLATION OF C.TRACHOMATIS}

To inoculate the specimens, the growth medium was removed from each of two vials with confluent monolayers and replaced by half of the transport medium $(1 \mathrm{ml})$. The vials were then centrifuged in a Mistral $6 \mathrm{~L}$ centrifuge at $2700 \mathrm{~g}$ for an hour at $35^{\circ} \mathrm{C}$. They were than incubated at $35^{\circ} \mathrm{C}$ for two days when the medium from one was removed, the monolayers fixed with methanol for 10 minutes, and then stained with Lugol's iodine for five minutes. The coverslips were removed from the vials, air-dried, and mounted in immersion oil. They were then examined at $\times \mathbf{4 0 0}$ magnification for typical chlamydial cytoplasmic inclusions. The duplicate vial was kept for further passage.

CHLAMYDIAL STRAIN TYPING

For strain typing the four-day mouse antisera method of Wang et al. (1973) was used. The sera were then typed according to the micro-immunofluorescence (MIF) test as described by Wang (1971). The antigens consisted of abundantly infected yolk sac suspensions. The original typed strains for the preparation of antigen were kindly supplied by Dr J. Treharne of the University of London and Dr C. Dawson of the WHO Chlamydia Reference Center in San Francisco.

ISOLATION OF N. GONORRHOEAE

Thayer-Martin selective culture medium was inoculated beside the patient and immediately incubated in a candle extinction jar at $36^{\circ} \mathrm{C}$. Identification of morphologically typical, oxidasepositive colonies was carried out by using the delayed fluorescent antibody technique with a commercially available antiserum (Difco). 


\section{Results}

ISOLATION OF C.TRACHOMATIS IN MEN WITH GONOCOCCAL AND NON-GONOCOCCAL URETHRITIS

The $\chi^{2}$ test and analysis of variance followed by the Scheffé method were used to analyse the results.

Altogether 139 men with gonococcal urethritis were examined for the presence of $C$. trachomatis. Positive cultures for this organism were obtained from $32(23 \%)$ of them (Table 1$)$.

Table 1 Incidence of $\mathrm{C}$. trachomatis infections in men with NGU and gonorrhoea

\begin{tabular}{lrrr}
\hline & & \multicolumn{2}{c}{ Chlamydia-positive } \\
\cline { 3 - 4 } & $N o$. & No. & $\%$ \\
\hline NGU & 238 & 124 & 52 \\
Gonorrhoea & 139 & 32 & 23 \\
Control group & 40 & 1 & $2 \cdot 5$ \\
\hline
\end{tabular}

All these patients with gonorrhoea were treated with a single dose of $2.5 \mathrm{~g}$ thiamphenicol by mouth and cure was confirmed in $93 \%$ by a negative smear and culture on a follow-up visit one week after treatment. Nineteen men whose gonorrhoea had been treated still had a urethral discharge at the follow-up visit although they had a negative culture for $N$. gonorrhoeae. These patients' condition fulfilled the criteria outlined and was diagnosed as PGU. Fifteen (79\%) of them initially harboured C. trachomatis, which could still be recovered at the time PGU was diagnosed.

We also followed-up 10 patients, who initially had a combined infection but who did not develop any sign of PGU after treatment. At the follow-up visit five $(50 \%)$ were still positive for $C$. trachomatis.

The effect of thiamphenicol as a single dose of $2.5 \mathrm{~g}$ on a genital infection with $C$. trachomatis can be assessed by the isolation rate after treatment in men with a proved infection. We obtained a negative culture result at the follow-up visit in seven men $(24 \%)$, whereas $22(76 \%)$ of a total of 29 patients still harboured Chlamydia one week after treatment.

The overall isolation rate of $C$. trachomatis in the group of 238 unselected patients with NGU was $52 \%$ (Table 1), three of these patients presented with urethritis associated with epididymitis and one patient had Reiter's disease. In 155 men with NGU specimens for $C$. trachomatis cultures were taken on their first visit and again about three days later on their second visit before treatment was initiated. Table 2 shows the recovery of $C$. trachomatis at each visit. We were able to re-examine 64 out of 116 men who had positive cultures on the initial visit. In eight $(11 \%)$ of these patients the repeat culture proved negative. Of the $122 \mathrm{men}$, who initially had a negative culture 91 were re-examined and eight $(9 \%)$ were found to be positive on that occasion.

Table 2 Recovery of C. trachomatis in patients with NGU on two consecutive visits

\begin{tabular}{|c|c|c|c|c|c|}
\hline \multirow{2}{*}{ Visit } & \multirow{2}{*}{$\begin{array}{l}\text { No. of } \\
\text { patients } \\
\text { examined }\end{array}$} & \multicolumn{2}{|c|}{$\begin{array}{l}\text { Chlamydia- } \\
\text { positive }\end{array}$} & \multicolumn{2}{|c|}{$\begin{array}{l}\text { Chlamydia- } \\
\text { negative }\end{array}$} \\
\hline & & No. & $\%$ & No. & $\%$ \\
\hline $\begin{array}{l}\text { First } \\
\text { Chlamydia-positive } \\
\text { Second }\end{array}$ & $\begin{array}{r}238 \\
64\end{array}$ & $\begin{array}{r}116 \\
56\end{array}$ & $\begin{array}{l}49 \\
89\end{array}$ & $\begin{array}{r}122 \\
8\end{array}$ & $\begin{array}{l}51 \\
11\end{array}$ \\
\hline Chlamydia-negative & 91 & 8 & 9 & 83 & 91 \\
\hline
\end{tabular}

Average interval between the first and second visit: $2 \cdot 7$ days

The duration of symptoms recorded at the initial visit in these eight patients was: $2,4,5,8,8,14,16$, and 16 days.

In the group of 40 controls, C. trachomatis was found in one of them (isolation rate $2.5 \%$ ).

SEROTYPES OF CHLAMYDIAL ISOLATES

We were able to establish the serotype according to the classification of Wang et al. (1973) from 35 chlamydial isolates recovered from 29 men and six women. The results of micro-IF typing are shown in Table 3.

Table 3 Results of serotyping chlamydial isolates by micro-IF test

\begin{tabular}{lllll}
\hline \multirow{3}{*}{ Total no. of isolates } & $C / J$ & $D / E$ & $F / G$ & $K$ \\
\cline { 2 - 5 } 35 & $1(2 \cdot 8 \%)$ & $18(51 \cdot 4 \%)$ & $10(28 \cdot 5 \%)$ & $6(17 \cdot 1 \%)$ \\
\hline
\end{tabular}

SOCIAL PROFILE AND CLINICAL

MANIFESTATIONS OF MEN WITH

GONORRHOEA, CHLAMYDIAL URETHRITIS, AND NSU

We compared the social profile and symptoms of men with gonorrhoea, Chlamydia-positive urethritis, and NSU. The profile of men with chlamydial urethritis by age (mean: 28 years), marital state (76\% single, $24 \%$ married), and percentage of homosexuals $(5 \%)$, was similar to the one established for gonorrhoea and NSU. The degree of promiscuity in the three groups, expressed by the number of recent sexual partners was also similar as shown in Table 4.

Table 4 Number of recent sexual partners of men with gonococcal, chlamydial, and non-specific urethritis

\begin{tabular}{llll}
\hline No. of partners & $\begin{array}{l}\text { Gonorrhoea } \\
(\%)\end{array}$ & $\begin{array}{l}\text { Chlamydial } \\
\text { urethritis } \\
(\%)\end{array}$ & $\begin{array}{l}\text { NSU } \\
(\%)\end{array}$ \\
\hline One & 36 & 41 & 48 \\
Two & 27 & 32 & 29 \\
Three or more & 37 & 27 & 22 \\
Total no. of patients & 90 & 91 & 85 \\
\hline
\end{tabular}


A history of previous episodes of urethritis, without a more precise definition, was obtained from $63 \%$ of patients with chlamydial urethritis and $60 \%$ with NSU, whereas only $41 \%$ of the men with gonorrhoea indicated previous genitourinary symptoms. In Table 5 the duration of urethral discharge is compared for the three groups. Most patients had symptoms for less than 14 days, but in each group there were several patients who had had symptoms for up to a year which made it necessary for a more accurate comparison to establish two groups ( $\leqslant 14$ days and $>14$ days).

Table 5 Comparison of clinical characteristics of gonococcal, chlamydial, and non-specific urethritis

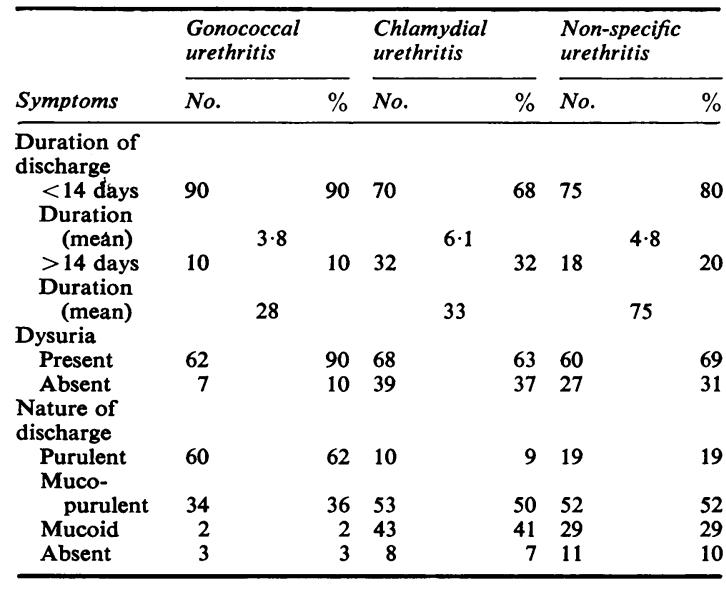

Dysuria was found significantly more often in patients with gonorrhoea $(90 \%)$ compared with Chlamydia-positive $(63 \%)$ and Chlamydia-negative $(69 \%)$ NGU $\left(\chi_{2}{ }^{2}=15.17 ; P<0.001\right)$. The incidence of this symptom in the two groups with NGU was similar. The urethral discharge was different in men with gonorrhoea, being mostly purulent $(62 \%)$ and mucopurulent $(36 \%)$ compared with NGU (Chlamydia-positive and Chlamydia-negative), which tended to be more mucoid and mucopurulent $\left(\chi_{4}{ }^{2}=88.86 ; \mathrm{P}<0.001\right)$. Table 5 shows the mean duration of discharge found in each group. Only the patients who had had a discharge for less than 14 days could be compared by statistical methods. The difference between the duration of discharge in gonorrhoea and NGU (Chlamydia-positive and Chlamydia-negative) was highly significant $(\mathrm{P}<0.01)$. On the other hand, the values obtained in chlamydial urethritis and NSU did not differ significantly $(P=0.06)$. In each group a few patients showed no urethral exudate on the first examination, although all of them gave a history of discharge during the days preceeding examination.

\section{Discussion}

We found a $C$. trachomatis isolation rate of $52 \%$ in our 238 patients with NGU. This incidence compares well with the recovery rates previously reported in this condition. Holmes et al. (1975) reported $42 \%$, Oriel et al. (1976) reported $48 \%$, Alani et al. (1977) and Schachter et al. (1975) obtained even higher isolation rates $-67 \%$ and $57 \%$, by only selecting cases of NGU with severe urethral discharge, which may correlate with a higher concentration of infectious elementary bodies in the exudate. An important question is the diagnostic yield of a single isolation attempt for $C$. trachomatis. To clarify this problem we repeated sampling, whenever possible, before initiating treatment. Two isolation attempts on separate visits at a mean interval of 2.7 days were performed in 155 patients with NGU. On the second examination we missed $11 \%$ of the chlamydial infections in the group of patients with positive cultures on the first visit. On the other hand, $9 \%$ of men with negative results to their first cultures proved positive on the second attempt. Thus we must assume, that with our present sampling and isolation techniques and no delay exceeding 24 hours before inoculation, we are missing at least $9 \%$ of chlamydial infections at any visit. It can be assumed that a third isolation attempt would provide an additional number of positive results. Some workers (Richmond et al., 1972; Oriel et al., 1976; Alani et al., 1977) found a highly significant relationship between the duration of symptoms and the rate of chlamydial isolation. This relationship could not be substantiated in our patients. The difference in duration of symptoms and time of sampling for cultures in our patients with Chlamydia-positive and Chlamydia-negative NGU was statistically not significant. Furthermore, in 91 out of 122 men with Chlamydia-negative NGU a second isolation attempt was performed, on an average of 2.7 days later. In only eight $(9 \%)$ of these men was the culture positive, of whom only three had had symptoms for less than six days. These findings indicate that in most men with NGU, chlamydial infections can be diagnosed within the first days after the onset of symptoms, and that it is unlikely that we are missing this organism in the group of men with NGU because sampling for culture is performed too early in the course of infection.

C. trachomatis was also recovered in $23 \%$ of patients with gonorrhoea. This rate is similar to the results reported in previous studies where $25 \%$ (Oriel et al., 1976) and 32\% (Richmond et al., 1972) of men with gonorrhoea were also infected with $C$. trachomatis. As all our patients with 
gonorrhoea were treated by a single dose of $2.5 \mathrm{~g}$ of thiamphenicol, we were able to obtain some information on the effect of this antibiotic on $C$. trachomatis. It appears that thiamphenicol, at the dosage used, has no effect or only a marginal one on $C$. trachomatis, as this could still be demonstrated at the follow-up visit in $76 \%$ of patients who had had a positive culture before treatment. Furthermore, the simultaneous infection with $C$. trachomatis and N. gonorrhoeae led to the development of PGU in $68 \%$ of these patients; an incidence of PGU of only $4.7 \%$ was observed in men without concomitant chlamydial infection. As Holmes et al. (1975) and Oriel et al. (1975) have indicated, this striking correlation between the presence of C. trachomatis and the development of PGU provides strong evidence in support of the pathogenicity of this agent in the urethra. Further evidence for this hypothesis is that $C$. trachomatis can be found only rarely in asymptomatic men. Schachter et al. (1975) reported the highest percentage of isolates $(7 \%)$ in a control group. We were able to discover only a single case in our control group of 40 men.

The results of immunotyping the chlamydial isolates are similar to the distribution of serotypes observed in England (Treharne et al., 1972) and in the USA (Holmes et al., 1975). The most prevalent serotypes in the genital tract belong to the group $\mathrm{D} / \mathrm{E}$, which we found in $51 \%$ of our typed isolates. The groups $\mathrm{F} / \mathrm{G}$ and $\mathrm{K}$ were also well represented, but $\mathrm{C} / \mathrm{J}$ was found only once. We were not able to detect any isolates belonging to types $\mathrm{H}$ and $\mathrm{I}$, which have also been recovered from the genital tract.

The social profile of men with gonorrhoea and NGU was very similar in our groups. An equivalent percentage $(63 \%$ and $60 \%)$ of men with chlamydial urethritis and NSU indicated that they had had previous episodes of genitourinary symptoms. This contrasts with the findings of Alani et al. (1977), who obtained higher isolation rates in men with no previous episodes of urethritis. Our data also showed that men with NGU and men with gonorrhoea generally present with a different history and different clinical manifestations, which may be used with the usual bacteriological techniques, to differentiate the two entities (Jacobs and Kraus, 1975). Unfortunately, these differences in symptoms do not appear as clearly when Chlamydiapositive and Chlamydia-negative NGU is compared. The duration of discharge before attendance was shortest in gonococcal urethritis $(3.8$ days), intermediate for NSU ( $4 \cdot 8$ days), and longest in chlamydial urethritis (6.1 days). However, the difference between the two groups with NGU is not significant. Dysuria was present in $90 \%$ of men with gonorrhoea, in $69 \%$ of men with NSU, and it was only slightly less common in men with chlamydial urethritis $(63 \%)$.

When the nature of the urethral discharge was compared, it appeared that gonorrhoea most often caused a purulent discharge, whereas chlamydial urethritis was generally characterised by a watery mucoid or mucopurulent discharge. The longer duration of symptoms, the presence of dysuria in little more than half of the patients, and the mucoid or mucopurulent urethral exudate confirm that the chlamydial infection of the male urethra generally runs a subacute and mildly symptomatic course, in contrast with a gonococcal infection, which causes a more acute and severe inflammation. The presence or absence of $C$. trachomatis in NGU, however, cannot be suspected on clinical grounds alone, as neither history nor clinical signs seems to differ sufficiently. We are grateful to $\mathrm{Dr}$ G. Van-Melle, Division d'Informatique, CHUV, Lausanne, for carrying out the statistical analysis.

\section{References}

Alani, M. D., Darougar, S., Burns, D. C. MacD., Thin, R. N., and Dunn, H. (1977). Isolation of Chlamydia trachomatis from the male urethra. British Journal of Venereal Diseases, 53, 88-92.

Department of Health and Social Security (1976). Report on the State of the Public Health for the Year 1975, pp. 47-51. HMSO: London.

Holmes, K. K., Handsfield, H. H., Wang, S. P., Wentworth, B. B., Turck, M., Anderson, J. B., and Alexander, E. R. (1975). Etiology of nongonococcal urethritis. New England Journal of Medicine, 292, 1199-1205.

Jacobs, N. F., and Kraus, S. J. (1975). Gonococcal and nongonococcal urethritis in men. Annals of Internal Medicine, 82, 7-12.

Juhlin, L. (1975). The situation of gonococcal and non-gonococcal infections in Sweden and other Scandinavian countries. In Genital Infections, pp. 25-28. Edited by D. Danielson, L. Juhlin, and P. A. Mardh. Burroughs Wellcome: Danderyd.

McChesney, J. A., Zedd, A., King, H., Russel, C. M., and Hendley, J. O. (1973). Acute urethritis in male college students. Journal of the American Medical Association, 226, 37-39.

Oriel, J. D., Reeve, P., Thomas, B. J., and Nicol, C. S. (1975). Infection with Chlamydia group $\mathbf{A}$ in men with urethritis due to Neisseria gonorrhoeae. Journal of Infectious Diseases, 131, 376-382.

Oriel, J. D., Reeve, P., Wright, J. T., and Owen, J. (1976). Chlamydial infection of the male urethra. British Journal of Venereal Diseases, $52,46-51$.

Perroud, H. (1977). Die Chlamydien-Urethritis. Schweizerische Rundschau für Medizin, 38, 1213-1217.

Reeve, P., Owen, J., and Oriel, J. D. (1975). Laboratory procedures for the isolation of Chlamydia trachomatis from the human genital tract. Journal of Clinical Pathology, 28, 910-914.

Richmond, S. J., Hilton, A. L., and Clarke, S. K. R. (1972). Chlamydial infection. Role of Chlamydia subgroup A in non-gonococcal and post-gonococcal urethritis. British Journal of Venereal Diseases, 48, 437-444.

Schachter, J., Hanna, L., Hill, E. C., Massad, S., Sheppard, C. W., Conte, J. E. Jr, Cohen, S. N., and Meyer, K. F., (1975). Are chlamydial infections the most prevalent venereal disease? Journal of the American Medical Association, 231, 1252-1255.

Treharne, J. D., Davev, S. J., Gray, S. J., and Jones, B. R. (1972). Immunological classification of TRIC agents and some recently isolated LGV agents by the micro-immunofluorescence test. British Journal of Venereal Diseases, 48, 18-25.

Wang, S. P. (1971). A micro-immunofluorescence method. Study of antibody response of TRIC organisms in mice. In Trachoma: Proceedings of an International Conference on Trachoma and Allied Diseases, Boston 1970, pp. 273-288. Edited by R. L. Nichols. Excerpta Medica: Amsterdam.

Wang, S. P., Kuo, C. C., and Graystone, J. T. (1973). A simplified method for immunological typing of trachoma-inclusion-conjunctivitis-lymphogranuloma-venereum organisms. Infection and Immunity, 7, 356-360. 